\title{
Abstracts from Nippon Eiseigaku Zasshi (Japanese Journal of Hygiene) vol. 67, no. 3
}

(C) The Japanese Society for Hygiene 2012

\section{Associations of Exposure to Dioxins and Polychlorinated Biphenyls with Diabetes: Based on Epidemiological Findings}

Nippon Eiseigaku Zasshi, 67, 363-374 (2012)

Hirokazu Uemura

Department of Preventive Medicine, Institute of Health Biosciences, the University of Tokushima Graduate School

Persistent organic pollutants (POPs) are a group of chemical substances that have the common properties of resistance to biodegradation, wide-range transportation, high lipophilicity, bioaccumulation in fat, and biomagnification in the food chain. POPs are persistent in the environment worldwide and have potential adverse impacts on human health and the environment. Polychlorinated dibenzo-p-dioxins (PCDDs), polychlorinated dibenzofurans (PCDFs), and polychlorinated biphenyls (PCBs) are well known chemicals that are considered as POPs.

The association between high-level exposure to dioxins and type 2 diabetes among U.S. Air Force veterans who had been exposed to Agent Orange contaminated with 2,3,7,8-tetrachlorodibenzo- $p$-dioxin (TCDD) during the Vietnam War was reported in the late 1990s. This association has been supported by similar epidemiologic studies, whose subjects were exposed to high doses of dioxins in their places of work involving phenoxyacid herbicide production and spraying, and in the industrial accident in Seveso, Italy.

Recently, low-level exposure to dioxins and PCBs has been reported to be linked to type 2 diabetes. Cross-sectional studies in the U.S. general population and Japanese general population showed that body burden levels of some dioxins and PCBs were strongly associated with the prevalence of type 2 diabetes. Very recently, following these cross-sectional studies, several prospective studies have suggested that low-level exposure to some PCBs predicted the future risk of type 2 diabetes in the general population. Environmental exposure to some dioxins and PCBs, which mainly accumulate in adipose tissue, may play a role in the development of type 2 diabetes.

\section{Regarding the Special Feature of "The Frontline of Nanoparticle Research": Progress of the Study Group on Fibrous and Particulate Substances (SGFPS)}

Nippon Eiseigaku Zasshi, 67, 375-382 (2012)

Takemi Otsuki ${ }^{1,2}$, Seishiro Hirano ${ }^{3,4}$

${ }^{1}$ Department of Hygiene, Kawasaki Medical School

${ }^{2}$ Chief Facilitator of the Study Group on Fibrous and Particulate

Substances (SGFPS) of Japanese Society of Hygiene

${ }^{3}$ Center for Environmental Risk Research, National Institute

for Environmental Studies

${ }^{4}$ Associate Facilitator of the Study Group on Fibrous

and Particulate Substances (SGFPS) of Japanese Society

of Hygiene

Among the symposia organized by various study groups in the Japanese Society of Hygiene (JSH), the Study Group on Fibrous and Particulate Substances (SGFPS) presented a symposium entitled "The frontline of nanoparticle research" chaired by Professor Takemi Otsuki (Kawasaki Medical School, Japan) and Dr. Seishiro Hirano (National Institute for Environmental Studies, Japan) on 26 March, 2012, as a part of the program of the 82nd Annual Meeting of JSH in Kyoto, Japan.

Special features consist of three presentations given at the abovementioned symposium. In this article, we introduce the progress of the Study Group on Fibrous and Particulate Substances (SGFPS) from the initial symposium entitled "Asbestos: Science and Society" held at the 76th Annual Meeting of JSH at Ube, Japan to the last abovementioned symposium in Kyoto. The health-related issues caused by exposure to fibrous materials such as asbestos and also particulated substances such as nanoparticles will be lasting in the future and researchers including our study group have to make their best efforts to resolve these problems and to reduce health impairments due to exposure to environmental fibrous and particulated substances. 


\section{Environmental Exposure to Silver and its Health Effects}

Nippon Eiseigaku Zasshi, 67, 383-389 (2012)

Takamitsu Miyayama ${ }^{1}$, Yuta Arai ${ }^{2}$, Seishiro Hirano ${ }^{1,2}$

${ }^{1}$ National Institute for Environmental Studies, Tsukuba, Ibaraki

${ }^{2}$ Graduate School of Pharmaceutical Sciences, Chiba University, Chiba

Silver (Ag) possesses a well-known antibacterial activity and has been used for medical treatment and cosmetics such as wound dressing and deodorant powders. Occupational Safety and Health Administration (OSHA) and Mine Safety and Health Administration (MSHA) proposed that the permissible exposure limit (PEL) for both metallic and most soluble Ag compounds should be $0.01 \mathrm{mg} / \mathrm{m}^{3}$. Argyria and argyrosis are known to be caused by deposition of insoluble Ag in the dermis and cornea/conjunctiva. However, the metabolic behavior and biological roles of Ag have not been well characterized in mammals. $\mathrm{Ag}$ can be absorbed into the systemic circulation from drinking water, and also through parenteral routes such as inhalation and dermal exposure. Experimental studies have demonstrated that $\mathrm{Ag}^{+}$induces and binds to metallothionein I and II (MTs), which are cysteine-rich proteins, in cells. MTs are major cytoplasmic metal binding proteins and thereby reduce cellular damage caused by toxic heavy metals including $\mathrm{Ag}$. Profiles of $\mathrm{Ag}$ distribution in MTs and other Agbinding proteins can be determined using high performance liquid chromatography-inductively coupled plasma mass spectrometry (HPLC-ICP-MS). This technique directly provides information on the intracellular behavior of $\mathrm{Ag}$, which is important for elucidating the mechanism underlying Ag toxicity. Silver nanoparticles (AgNPs) are also commercially used mainly as antimicrobial agents. Despite the widespread use of AgNPs, relatively few studies have been undertaken to evaluate the health effects of AgNP exposure. In the present paper, we discuss the absorption, toxicodynamics, and metabolism of both $\mathrm{Ag}$ and AgNPs in mammals and their health effects.

\section{Significance of Comprehensive Gene Expression Analysis for Evaluation of Biological Effects of Manufactured Nanomaterials}

Nippon Eiseigaku Zasshi, 67, 390-395 (2012)

Katsuhide Fujita ${ }^{1,2}$, Makiko Fukuda ${ }^{2}$, Hitoshi Iwahashi ${ }^{3}$

${ }^{1}$ Research Institute of Science for Safety and Sustainability (RISS), National Institute of Advanced Industrial Science and Technology (AIST)

${ }^{2}$ Technology Research Association for Single Wall Carbon Nanotubes (TASC)

${ }^{3}$ Faculty of Applied Biological Sciences, Gifu University

The industrial applications of manufactured nanomaterials (MNs) are expected to be extended to next-generation devices. On the other hand, concern over the effects of MNs on human health has risen owing to advances in the development of nanotechnology. Indeed, little is known about the mechanism of action of MNs. The New Energy and Industrial Technology Development Organization of Japan (NEDO) launched a new research project entitled "Evaluating risks associated with manufactured nanomaterials (P10024)" in 2006. The project demonstrated no adverse effects of MN inhalation exposure on the rat lungs, as determined by histopathological examination and bronchoalveolar lavage (BAL) fluid analysis. In parallel with this research, we have performed comparative gene expression analysis using DNA microarrays in rat lungs after inhalation exposure (4 weeks, $6 \mathrm{~h}$ a day, 5 days a week) to single-wall nanotubes (SWCNTs), multiwall nanotubes (MWCNTs), $\mathrm{C}_{60}$ fullerene and ultrafine nickel oxide particles (Uf-NiO) as reference materials for the purpose of gaining insights into the molecular events following the exposure. In this review, we introduce an outline of the project, and discuss about the significance of comparative gene expression analysis for evaluation of the biological effects of MNs.

\section{Pulmonary Toxicity of Manufactured Nanomaterials-Report of NEDO Project}

Nippon Eiseigaku Zasshi, 67, 396-400 (2012)

Yasuo Morimoto

Department of Occupational Pneumology,

University of Occupational and Environmental Health, Japan

We introduced the report on the pulmonary toxicity of manufactured nanomaterials as a national project in Japan conducted by the New Energy and Industrial Technology Development Organization (NEDO project). Well-characterized and well-dispersed industrial nanomaterials were used in this project. Using multiwall carbon nanotubes (MWCNTs), we conducted intratracheal instillation and inhalation studies. At a low dose $(0.2 \mathrm{mg} / \mathrm{rat})$ and a high dose $(1 \mathrm{mg} /$ rat) of almost individual MWCNTs, transient and persistent inflammatory responses were induced in the lungs following intratracheal instillation. Well-dispersed MWCNTs at a low concentration $\left(0.37 \mathrm{mg} / \mathrm{m}^{3}\right)$ did not induce pulmonary inflammation. Using singlewall carbon nanotubes (SWCNTs), we also conducted both studies. At low $(0.2 \mathrm{mg} / \mathrm{rat})$ and high doses $(0.4 \mathrm{mg} / \mathrm{rat})$ of well-dispersed SWCNTs, persistent inflammatory responses were induced in the lungs following intratracheal instillation. Well-dispersed MWCNTs at low and high concentrations ( 0.03 and $0.13 \mathrm{mg} / \mathrm{m}^{3}$, respectively) did not induce pulmonary inflammation. On the basis of these data from mainly inhalation studies, the acceptable exposure concentration of carbon nanotubes is proposed to be $0.03 \mathrm{mg} / \mathrm{m}^{3}$ in Japan.

\section{Activities in Daily Life and Changes in Care Level among Users of Preventive Care Service under Long-Term Care Insurance}

Nippon Eiseigaku Zasshi, 67, 401-407 (2012)

Toshimasa Sone ${ }^{1,2}$, Naoki Nakaya ${ }^{3}$, Yasutake Tomata ${ }^{1}$, Jun Aida ${ }^{4}$, Ichiro Okubo ${ }^{5}$, Satoko Ohara ${ }^{6}$, Shuichi Obuchi ${ }^{7}$, Michiko Sugiyama ${ }^{8}$, Seiji Yasumura ${ }^{9}$, Takao Suzuki ${ }^{7,10}$, Ichiro Tsuji ${ }^{1}$

${ }^{1}$ Tohoku University Graduate School of Medicine

${ }^{2}$ Tohoku Fukushi University

${ }^{3}$ Kamakura Women's University

${ }^{4}$ Tohoku University Graduate School of Dentistry

${ }^{5}$ University of Tsukuba Graduate School of Comprehensive Human

Sciences

${ }^{6}$ Tokyo Medical and Dental University, Dental Hospital

${ }^{7}$ Tokyo Metropolitan Institute of Gerontology

${ }^{8}$ Kanagawa University of Human Services

${ }^{9}$ Fukushima Medical University

${ }^{10}$ National Institute for Longevity Sciences

Objectives: The aim of this study was to investigate the association between social roles (engaged in working outside or house work) or hobbies and changes in care level among users of Preventive Care Service under Long-Term Care Insurance. 
Methods: A total of 8,734 Preventive Care Service users were analyzed. The assessment was conducted between February 2007 and December 2008. The improvement, maintenance, and aggravation in care level were defined by changes in care level from the beginning to the end. To assess their activities in daily life, the participants were asked, "Which is the major activity in your daily life? Please select one from the following: working outside, house work, hobbies, watching television, others, or none." We used the multiple logistic regression model to calculate the odds ratio (OR) and $95 \%$ confidence interval (CI) of the maintenance or improvement in care level according to the category of activity.

Results: As compared with subjects who answered none, the multivariate adjusted ORs $(95 \% \mathrm{CI})$ of the maintenance or improvement in care level were $2.0(1.4-2.9)$ for those who answered working outside, $1.5(1.2-1.8)$ for those who answered house work, and 1.5 (1.2-1.9) for those who answered hobbies. However, those who answered watching television or others did not show a significant association with the maintenance or improvement in care level.

Conclusions: In this study, compared with subjects who answered none, those who answered that they were engaged in working outside, house work, or hobbies were associated with having significantly higher ORs of the maintenance or improvement in care level.

\section{Examination of Influence of Adolescent Lifestyle on Oral Hygiene}

Nippon Eiseigaku Zasshi, 67, 408-416 (2012)

Kimiko Sato

Prefectural University of Hiroshima

Objectives: In the "biopsychosocial model" propounded by Engel, psychological, biological, and social factors are considered to be interrelated contributors to the causes of illnesses. In this study, we investigated the relationship of oral health in Japanese juveniles with psychological, biological, and social factors.

Methods: The subjects were 227 junior high school students who belonged to a relatively small junior high school in a city, Hiroshima prefecture, Japan. The investigation period was from October 2010 to May 2011. The status of oral health was determined from the results of the caries risk test and periodontal examinations performed using saliva samples. In addition, subjective oral symptoms were determined using questionnaires. Thereafter, the interrelationships among psychological, biological, and individual social (mainly status in the family) factors and preventive methods against oral diseases were discussed.

Results: A large number of students were identified to have a high caries risk, and $20 \%$ of the students with periodontitis were in need of medical treatment. Oral health status was not influenced equally by the biological, psychological, and social factors. The results suggest that individual social factors influence biological changes through a psychological factor interaction.

Conclusions: The findings suggest that oral disease prevention in juveniles requires an active positive influence of individual social factors, that is, relationships with family members and other individuals, such as medical and educational professionals and individuals involved in juvenile oral health improvement programs.

\section{Relationship between Baroreflex Function and Training Effects on Altitude Training}

Nippon Eiseigaku Zasshi, 67, 417-422 (2012)

Ryo Yanagida ${ }^{1}$, Yojiro Ogawa ${ }^{1}$, Fumio Mizuochi ${ }^{2}$, Tsukasa Suzuki ${ }^{3}$, Masanori Takahashi ${ }^{2}$, Kenichi Iwasaki ${ }^{1}$

${ }^{1}$ Division of Hygiene, Department of Social Medicine, Nihon

University School of Medicine

${ }^{2}$ Nihon University College of Humanities and Sciences

${ }^{3}$ Nihon University School of Dentistry at Matsudo

Objective: Altitude training is frequently used for athletes requiring competitive endurance in an attempt to improve their sea-level performance. However, there has been no study in which the mechanisms by which spontaneous arterial-cardiac baroreflex function changes was examined in responders or nonresponders of altitude training. The purpose of this study was to clarify the different effects of altitude training on baroreflex function between responders and nonresponders.

Methods: Twelve university student cross-country skiers (6 men, 6 women; age, $19 \pm 1$ years) participated in the altitude training in a camp for 3 weeks, which was carried out in accordance with the method of Living High-Training Low. Baroreflex function was estimated by transfer function analysis before and after the training. Results: The responders of the training were 3 men and 2 women, and the nonresponders were 3 men and 4 women. In the responders, the transfer function gain in the high-frequency range significantly increased after the training $(28.9-46.5 \mathrm{~ms} / \mathrm{mmHg}, p=0.021)$. On the other hand, no significant change in this index was observed in the nonresponders $(25.9-21.2 \mathrm{~ms} / \mathrm{mmHg}, p=0.405)$.

Conclusion: As indicated by the results of transfer function gain in the high-frequency range, the baroreflex function in the responders increased significantly after the altitude training, whereas no significant change was observed in the nonresponders.

\section{Determination of Tobacco-Specific $N^{\prime}$-Nitrosamines in Mainstream Smoke from Japanese Cigarettes}

Nippon Eiseigaku Zasshi, 67, 423-430 (2012)

Koichi Sugiyama $^{1,2}$, Yohei Inaba ${ }^{2}$, Tadamichi Ohkubo ${ }^{2}$, Shigehisa Uchiyama ${ }^{2}$, Yukihiko Takagi ${ }^{1}$, Naoki Kunugita ${ }^{2}$

${ }^{1}$ Department of Veterinary Medicine, Azabu University

${ }^{2}$ Department of Environmental Health, National Institute of Public Health

Objectives: Mainstream smoke from cigarettes contains tobaccospecific $N^{\prime}$-nitrosamines (TSNAs) listed as Group 1 and 3 carcinogens by the International Agency for Research on Cancer (IARC). Herein, we report on a method of measuring the concentrations of TSNAs in mainstream smoke from the ten top-selling Japanese cigarette brands using an ISO regime by International Organization for Standardization (ISO) and HCI regime of Health Canada.

Methods: Tar in mainstream smoke was collected on a Cambridge filter pad using a smoking machine. The filter pad was immersed in 40 $\mathrm{mL}$ of ammonium acetate $(\mathrm{pH}$ 6.8) and shaken for $30 \mathrm{~min}$. The extract was then loaded into a C18 column. After washing with $5 \mathrm{~mL}$ of $10 \%$ methanol and eluting with $5 \mathrm{~mL}$ of $70 \%$ methanol, the eluate was concentrated to $1 \mathrm{~mL}$ for LC-MS/MS analysis. 
Results: The concentrations of TSNAs in all cigarette brands were higher when determined using the HCI regime than when determined using the ISO regime. Furthermore, the concentrations of TSNAs measured using both the ISO and HCI regimes showed negligible correlation to the tar and nicotine concentrations indicated on package labels. The cigarette samples used in the study were categorized into four classes: ultralow-, low-, medium-, and high-yield brands, which corresponded to $1,3-6,8-10$, and $14 \mathrm{mg}$ tar/cigarette, respectively.
The concentration of TSNAs in ultralow-yield cigarettes was $210 \mathrm{ng} /$ cigarette, as measured using the HCI regime, which was nearly equal to that in high-yield cigarettes (180 ng/cigarette).

Conclusions: Exposure to TSNAs from mainstream smoke from ultralow-yield cigarettes is comparable to that from high-yield cigarettes. To properly evaluate the risk of smoking, not only the concentrations of tar and nicotine but also those of other chemicals, including TSNAs, should be printed on package labels. 